\title{
SENSORES DE BAJO COSTO PARA EL MONITOREO DE CALIDAD DEL AIRE
}

\author{
Low cost sensors for air quality measurement
}

EPISTEMUS

ISSN: 2007-8196 (electrónico) ISSN: 2007-4530 (impresa)

\section{Gilberto García Navarrete ${ }^{1}$ Kenia Gpe. Rico Soto ${ }^{2}$}

Recibido: 12 de septiembre de 2019, Aceptado: 30 de noviembre del 2019

Autor de Correspondencia: M.I. Gilberto García Navarrete Correo: gilberto.garcia@unison.mx

\section{Resumen}

La tecnología cada vez se perfecciona con dispositivos compactos y baratos que sustituyan a los equipos actuales en diferentes áreas, los sensores de bajo costo presentan desde 2013 una alternativa viable como sustituto de las tecnologías actuales en monitoreo de calidad del aire. En el marco actual de su uso se cuenta con disposición de estos dispositivos en el mercado y con gran variedad de principios de operación por lo que la estandarización y calibración de su aplicación aún se encuentra en desarrollo. Las variables de selección dependerán de la variable de interés y se vuelve fácil de adaptarse a lo comercialmente disponible. El funcionamiento, principios de operación, así como las ventajas y desventajas de esta tecnología se presentan en el desarrollo del artículo.

Palabras clave: Sensores de bajo costo, calidad del aire, contaminante criterio.

\section{Abstract}

Technology is increasingly perfected with compact and cheap device that replace currente equipamente in different áreas, the low-cost sensors present since 2013 a viable alternative as a sustitute for current technologies in aire quality monitoring. In the current framework of its use, these device are available in the market and with a wide variety of operating principles, so the standardization and calibration of their application is still under development. The selection variables will depend on the cariable of interest and it becomes easy to adapt to what is commercially available. The operation, principles of operation, as well as the advantages and disadvantages of this technology are presented in the development of the article.

Keywords: Low-cost sensors, air quality, pollutant criteria. 


\section{INTRODUCCIÓN}

La definición de sensores de bajo costo (SBC) varía un poco dependiendo del autor. Los SBC como su nombre lo indica tienen la particularidad de que su precio debe ser inferior a un valor máximo, este límite puede variar entre los $\$ 100.00$ y los $\$ 500.00$ dólares [1].

Los SBC son una gama de sensores que sus ventajas de precio y tamaño, los posiciona como los más usados en el mercado actual en diferentes áreas de la industria y más recientemente en el área de monitoreo de calidad del aire. Otras ventajas significativas son los costos de operación y mantenimiento involucrados, además de tener la facultad de presentar información en tiempo real generando datos en volumen suficiente y disponibles en todo momento. Por otra parte, al ser una tecnología que se encuentra aun en desarrollo en el área de calidad del aire, presenta como sus principales desventajas la falta de estandarización de principios y validación de información.

En el presente artículo se presentan los principios generales de operación de los sensores aplicados en el área de calidad del aire, así como las ventajas y desventajas de estos.

\section{ANTECEDENTES}

En un principio los SBC se desarrollaron para monitorear la calidad del aire en el interior de casas habitación. El 2013 la EPA (Environmental Protection Agency) llevo a cabo un taller para el desarrollo de SBC en el cual se planteaban los avances y la aplicación de estos dispositivos compactos que permitirían la generación de información sobre la calidad del aire. La diversidad de principios, la comunicación en línea de los dispositivos y la falta de estándares en calibración representaban el principal objetivo de este evento [1].

Para el 2015, se comienza el desarrollo de un proyecto para la conformación de una estación de monitoreo con este tipo de sensores (Figura 1), probando la efectividad de diferentes modelos de sensores de casi todos los contaminantes criterio, lo cual permitió probar la

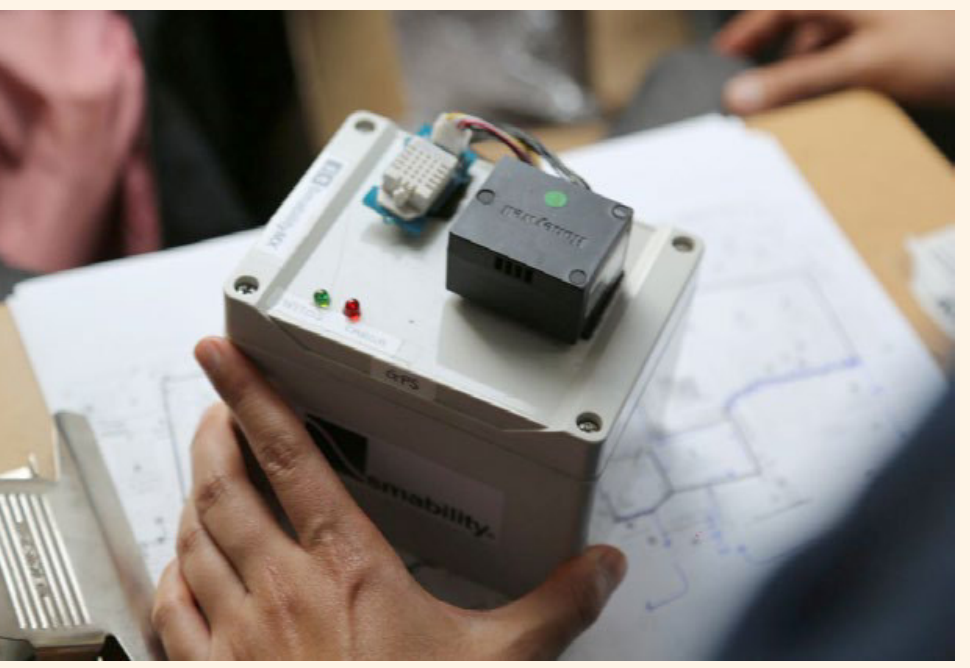

estabilidad del equipo en línea con un planteamiento de desarrollo de dos años. Los resultados de interferencia entre los sensores de diferentes variables es la información más relevante que se obtuvo de este estudio [2].
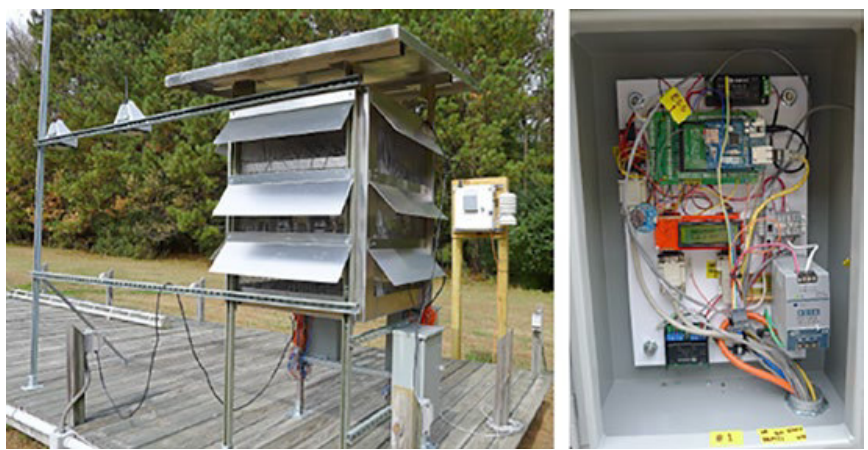

Figura 1. Estación de prueba del estudio de la EPA de 2015 [2].

En 2017 se publicó el artículo de un proyecto realizado en la ciudad de Lima Perú, cuyas variables principales fueron el $\mathrm{SO}_{2}$ y el $\mathrm{CO}_{2}$, utilizando $\mathrm{SBC}$, el cual se centraba en la búsqueda de un modelo para la predicción de las concentraciones mediante la aplicación de algoritmos de redes neuronales como la conexión entre las diferentes estaciones móviles colocadas. Cabe mencionar que el estudio no obtuvo resultados exitosos, sus predicciones fueron muy alejadas de las reales [3].

La variable más popular para la aplicación de esta tecnología es el material particulado, como el caso de PST con los detectores de humo, pero en especial las partículas $\mathrm{PM}_{10^{\prime}} \mathrm{PM}_{2.5}$ y $\mathrm{PM}_{1.0}$.

Posteriormente en el 2018, la EPA se encuentra con la aplicación de un proyecto de la mano de AirMapear para el uso de un monitoreador compacto móvil (similar al tamaño de una hielera pequeña) para la detección de material particulado. Durante el mismo año en España se desarrolló una tesis para la creación de un módulo de detección de $\mathrm{PM}_{10}$ y $\mathrm{PM}_{2.5}$, enfocada al desarrollo de un sensor para estas variables, a la selección del tipo de hardware y la comunicación con Arduino a un teléfono celular con Android como sistema operativo [4].

A finales de 2018 varios países latinoamericanos se dedicaron al desarrollo de sus propios SBC tal es el caso de Colombia y Perú.

\section{VARIABLES DE INTERÉS}

Para el monitoreo de calidad de aire se requiere que se consideren dos tipos de variables, las variables meteorológicas y las variables propiamente de calidad del aire (concentración/presencia de contaminantes). Los SBC disponibles en el mercado nos permiten monitorear ambos tipos, sin embargo, en el caso de aquellos que recolectan datos de la concentración de los contaminantes nos limitan en el rango de detección y en la sensibilidad del cambio de estos. 


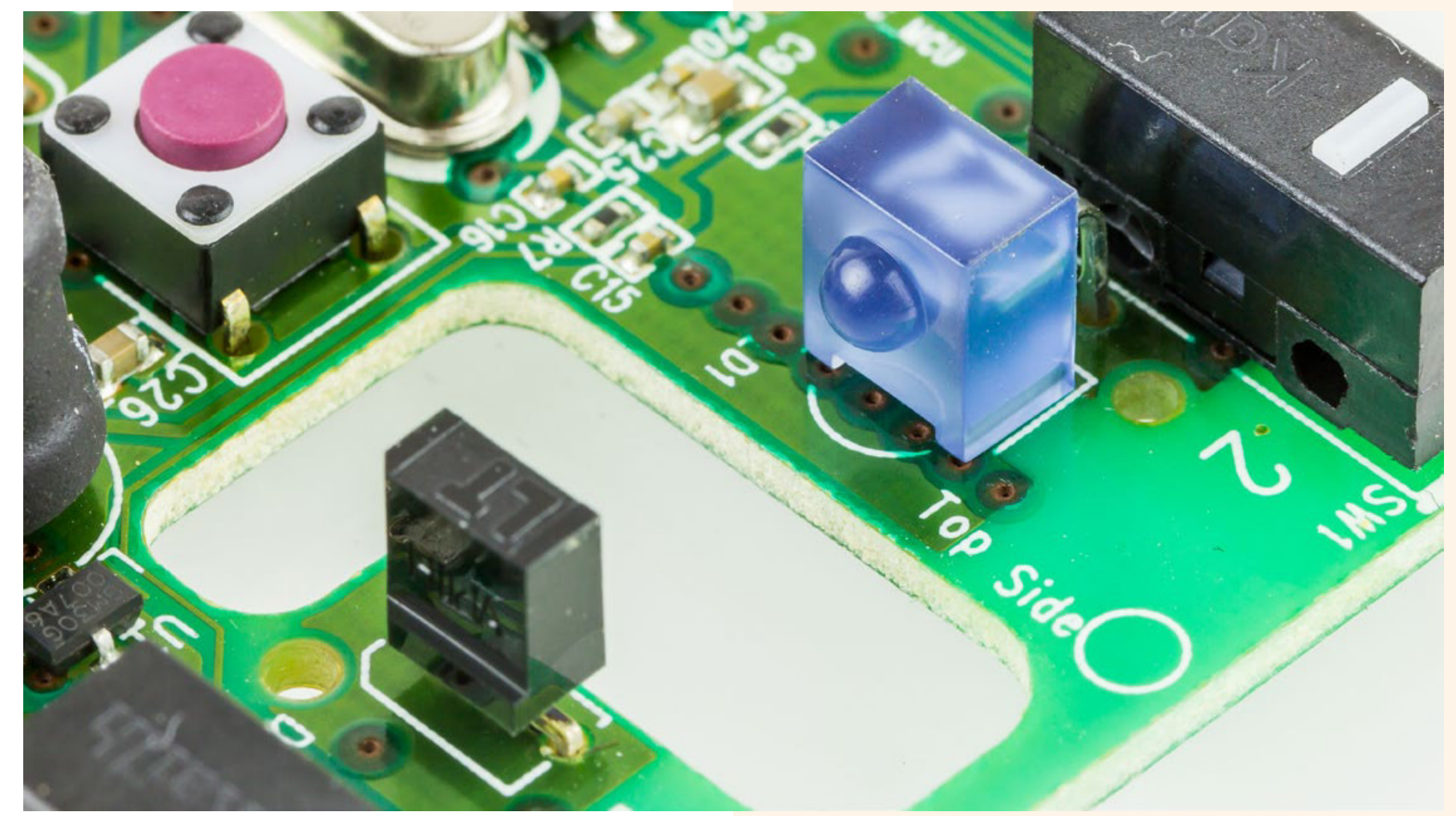

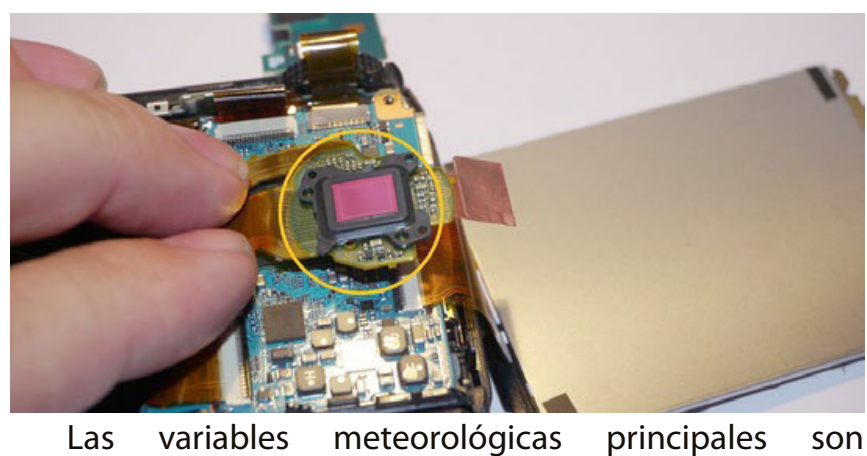

temperatura, humedad relativa y presión barométrica, esto debido a que son auxiliares en las correcciones necesarias para las variables de calidad del aire debido a que la mayoría de estas son gases. También se pueden agregar como variables de este tipo la velocidad y dirección del viento, radiación UV y lluvia.

Las variables de calidad del aire son aquellas que involucran a los contaminantes criterio (que incluyen en el caso de México 6 de los 7 normados), que son la concentración del ozono $\left(\mathrm{O}_{3}\right)$, el monóxido de carbono (CO), el bióxido de azufre $\left(\mathrm{SO}_{2}\right)$, el bióxido de nitrógeno $\left(\mathrm{NO}_{2}\right)$ y las partículas suspendidas menores a 10 y a 2.5 micrómetros $\left(\mathrm{PM}_{10}\right.$ y $\left.\mathrm{PM}_{2.5}\right)$.

Para la selección de los sensores es necesario conocer el rango en el que se requiere manejar dichos sensores, esto debido a que en el mercado se cuenta con varios SBC con diferentes rangos de concentraciones y sensibilidades que no son útiles para el área de calidad del aire. Los límites permisibles normados en los países nos presentan la principal variable de selección para los SBC en monitoreo de calidad del aire.

\section{En la Tabla 1 se muestran los límites permisibles de la normatividad mexicana para cada contaminante criterio.}

\begin{tabular}{|c|c|c|c|}
\hline \multirow{2}{*}{ Contaminante } & \multicolumn{2}{|c|}{ Concentración } & \multirow{2}{*}{ Tiempo } \\
\hline & (ppm) & $\left(\mu \mathrm{g} / \mathrm{m}^{3}\right)$ & \\
\hline \multirow{2}{*}{ Jzono $\left(\mathrm{O}_{3}\right)[5]$} & 0.095 & & $1 \mathrm{hr}$ \\
\hline & 0.070 & & $8 \mathrm{hrs}$ \\
\hline $\begin{array}{l}\text { Monóxido de Carbono } \\
\text { CO) [6] }\end{array}$ & 11 & 12,595 & $\begin{array}{l}8 \mathrm{hrs} \\
\text { (móvil) }\end{array}$ \\
\hline \multirow{3}{*}{$\begin{array}{l}\text { Dióxido de azufre }\left(\mathrm{SO}_{2}\right) \\
\text { 7] }\end{array}$} & 0.200 & 524 & $8 \mathrm{hrs}$ \\
\hline & 0.110 & 228 & $24 \mathrm{hrs}$ \\
\hline & 0.025 & 66 & Anual \\
\hline $\begin{array}{l}\text { Dióxido de Nitrógeno } \\
\left(\mathrm{NO}_{2}\right)[8]\end{array}$ & 0.021 & 395 & $1 \mathrm{hr}$ \\
\hline $\begin{array}{l}\text { Jartículas suspendidas } \\
\text { otales (PST) [9] }\end{array}$ & $\mathrm{n} / \mathrm{a}$ & 210 & $24 \mathrm{hrs}$ \\
\hline \multirow{2}{*}{$\mathrm{M}_{10}[9]$} & $\mathrm{n} / \mathrm{a}$ & 75 & $24 \mathrm{hrs}$ \\
\hline & $\mathrm{n} / \mathrm{a}$ & 40 & Anual \\
\hline \multirow{2}{*}{$\mathrm{M}_{2.5}[9]$} & $\mathrm{n} / \mathrm{a}$ & 45 & $24 \mathrm{hrs}$ \\
\hline & $\mathrm{n} / \mathrm{a}$ & 12 & Anual \\
\hline
\end{tabular}




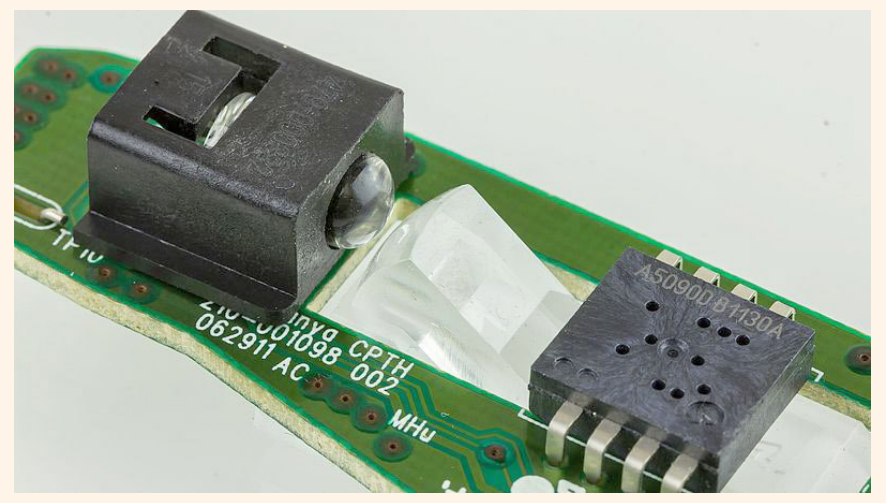

PRINCIPIOS DE OPERACIÓN DE LOS SBC

Basándonos en la información química que genera el sensor y las características del transductor que lo conforma es posible clasificarlos en tres tipos, electroquímicos, piezoeléctricos y ópticos [10].

\section{Sensores piezoeléctricos}

El funcionamiento de estos sensores se basa en la micro-gravimetría, registra los cambios de masa que se dan como característica de la interacción de las diferentes especies químicas con el sensor. De manera general se forman con material piezoeléctrico que es sometido a oscilación [10].

\section{Sensores electroquímicos}

Los sensores de este tipo se pueden clasificar en tres subtipos, potenciométricos, voltimétricos y conductimétricos. Esta clasificación se da por el mecanismo que presenta cada sensor y el principio físico de operación. [10].

Potenciométricos: presenta información de la relación explicita entre el potencial de un electrodo indicador/ contador y la concentración de la especie objetivo. Como no es posible medir el potencial de una sola fase, se requiere introducir un segundo electrodo (electrodo de referencia) para medir la diferencia de potencial entre el electrodo indicador y el de referencia. Estos sensores se pueden subdividir conforme a la base de funcionamiento del electrodo que utilizan en dos tipos [10]:

- Sensores basados en electrodos selectivos de iones conocidos como ISE (por sus siglas en ingles de lon Selective Electrodes).

- Sensores basados en transistores de efecto de campo.

Voltimétricos: Su principio de operación es la relación corriente-voltaje, se aplica potencial al sensor y se mide una corriente proporcional a la especie electroactiva de interés, como caso especial este tipo de sensor tiene el sensor amperométrico, al cual se aplica un voltaje constante. El mecanismo de funcionamiento se presenta al reaccionar el sensor con la sustancia objetivo (gas para este caso) que genera una señal eléctrica proporcional a la concentración del gas presente. [10].
Conductimétricos: Se basan en la medición de la conductividad a una serie de frecuencias, registran los cambios de la conductividad eléctrica de una capa o la mayor parte de un material, cambios que se dan debido a la presencia de la sustancia objetivo. [10].

\section{Sensores ópticos}

Este tipo de sensores tienen como principio de operación la detección de un haz de luz u otras ondas electromagnéticas mientras se encuentra en interacción con las especies químicas de interés. Los arreglos y principios físicos de los sensores ópticos para la detección de gases son bastantes, al igual que los métodos de detección de señal tales como los detectores infrarrojos (opera en el espectro infrarrojo) y la fotoionización (opera en el espectro ultravioleta) [10].

\section{DISPONIBILIDAD EN EL MERCADO}

Los SBC disponibles en el mercado se pueden encontrar como únicamente el sensor o en forma modular (con tarjeta de comunicación), usualmente los que cuentan con forma modular pueden detectar más de una variable como en el caso de los sensores de humedad relativa que comúnmente pueden también detectar temperatura y los sensores de gases que suelen tener sensibilidad a detectar más de un solo tipo de gas.

De forma general los sensores comerciales cuentan con limitaciones de fábrica, como lo son los rangos de operación, temperatura y detección específicos, así como con sensibilidades de detección que no resultan las óptimas para el monitoreo de calidad de aire, pero como

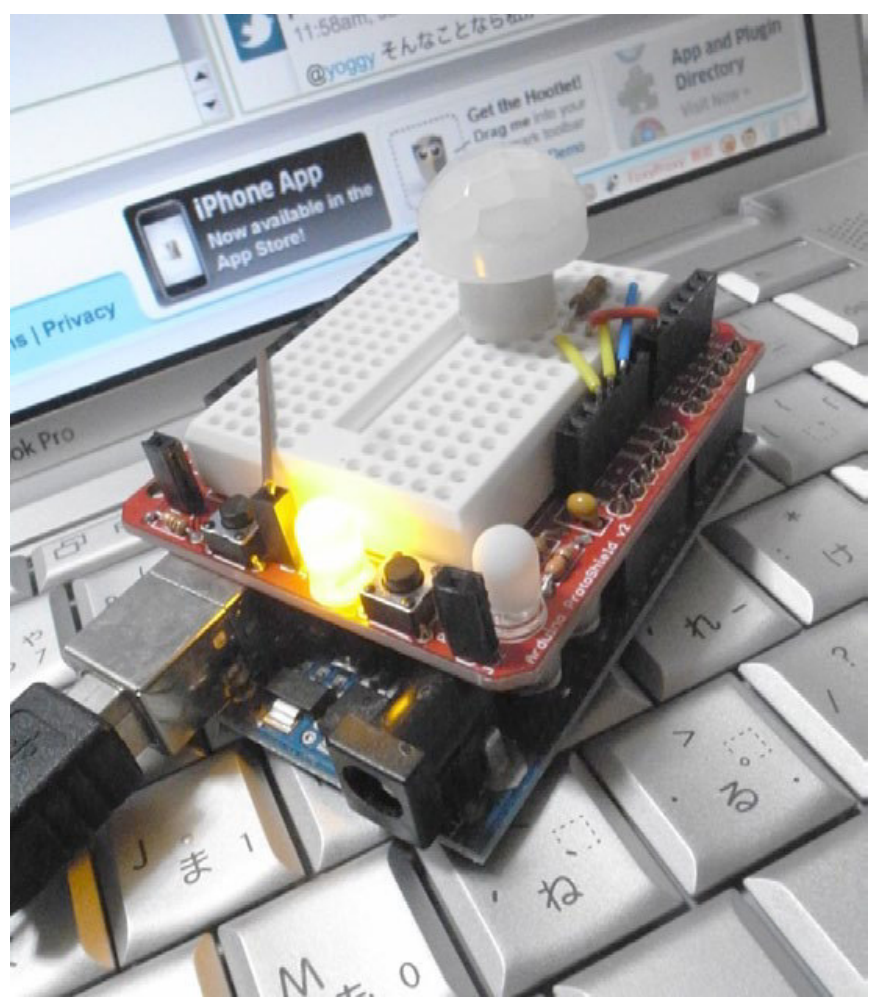




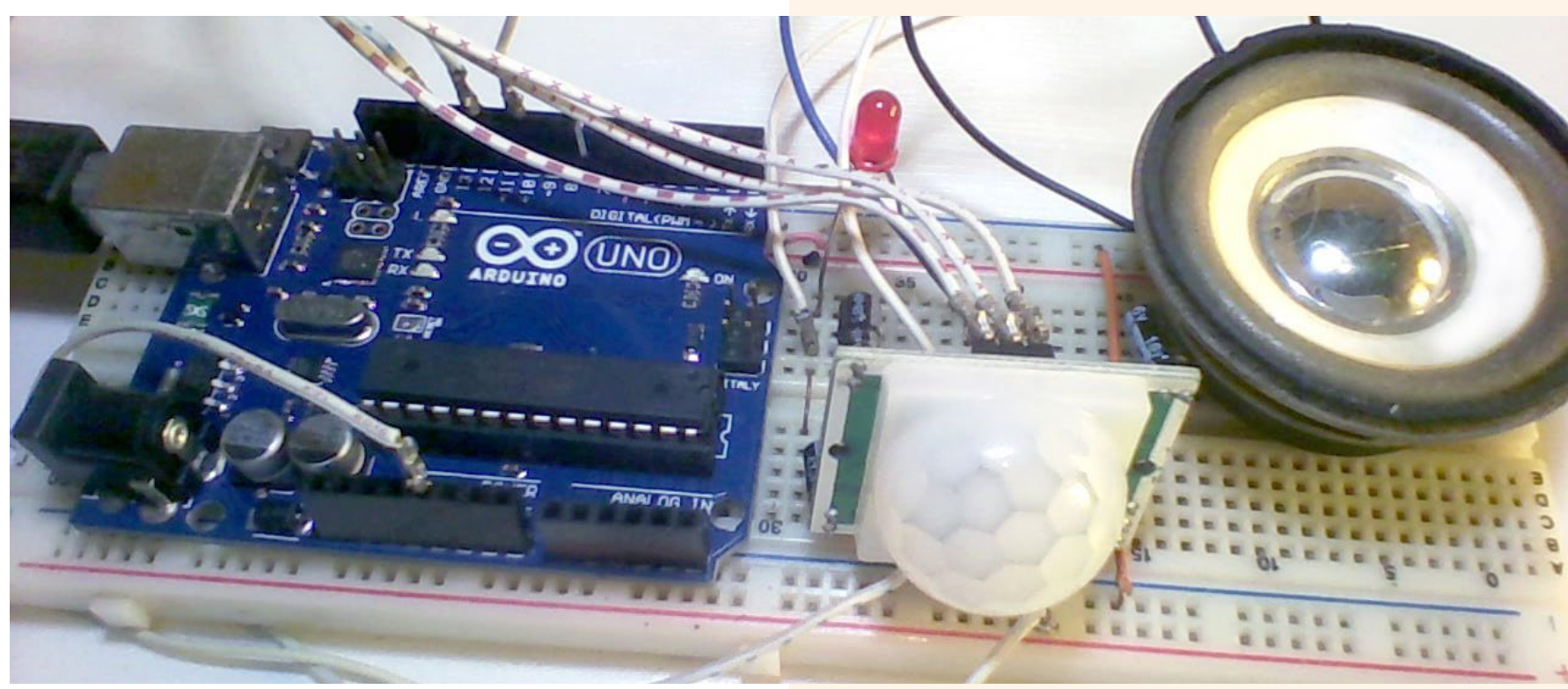

se mencionó antes se encuentran en constante desarrollo dispositivos que puedan cumplir con los parámetros deseados.

En el caso particular de los SBC para gases se cuenta con "interferencias cruzadas", esto significa que teniendo un sensor para un gas particular como el ozono el que se encuentre presencia de otro gas por ejemplo el $\mathrm{CO}$ puede generar un error mayor en la lectura tomada por el sensor para el gas objetivo, en este ejemplo el ozono. Por otra parte, la sensibilidad menor en los sensores de gas al mostrar concentraciones es de 20 ppb, lo que implica que no es posible monitorear de forma exacta si se encuentra dentro del valor máximo permisible dado que la mayoría de estos se presentan con números impares en esta unidad.

Los precios varían dependiendo del tipo de sensor, los más baratos son los de temperatura llegando a costar hasta centavos de dólar comprados por mayoreo, los sensores para gases se encuentran con precios que van desde 5.75 USD, hasta los $\$ 75.00$ USD en su versión modular.

En las Figuras de la 2 y 3 se muestran modelos de algunos de los sensores disponibles en el mercado, para las diferentes variables de interés.

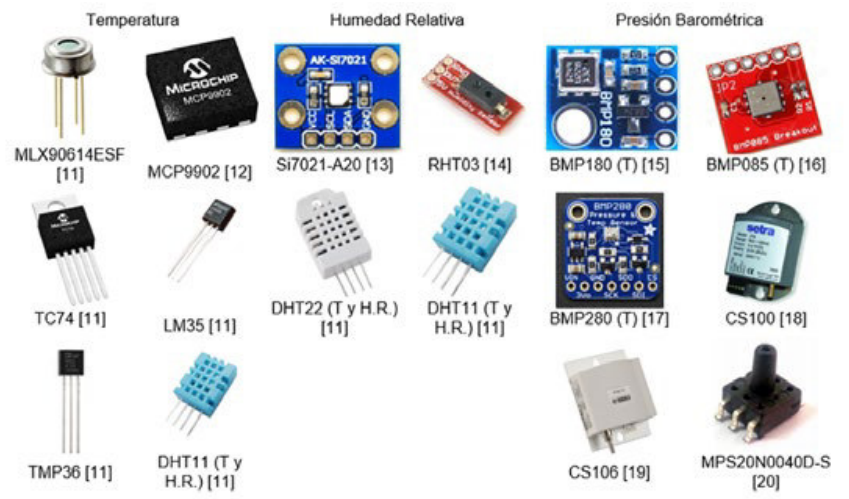

Figura 2. SBC disponibles en el mercado para variables meteorológicas.

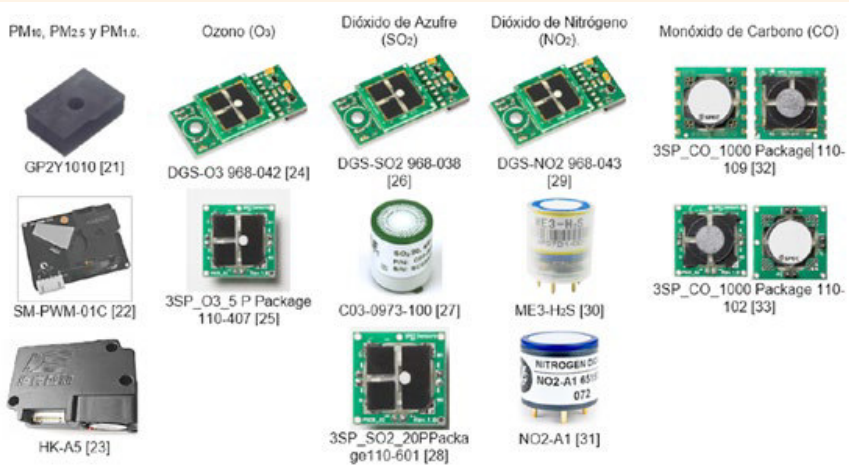

Figura 3. SBC disponibles en el mercado para variables de calidad del aire.

\section{VENTAJAS Y DESVENTAJAS DEL USO DE SBC}

Las ventajas de esta tecnología son obvias menor costo del equipo, así como de operación y mantenimiento, comparada con la tecnología actual en el área de calidad del aire.

La comunicación en tiempo real, que permite la acción de correcciones inmediatas; la generación de datos en volumen, que permite el desarrollo de programas de prevención y contingencia, asi como de modelos de proyección.

La facilidad de difusión de los datos generados a todos los interesados permite tener un control de exposición personal relacionándolo directamente con la prevención de salud individualizada.

Entre sus principales desventajas tenemos que aun no se cuenta con protocolos de calibración y operación estandarizados. En el caso de ciudades con climas extremosos la limitante de los rangos de operación de temperatura son un reto a resolver dado que la mayoría de los sensores disponibles comercialmente solo alcanzan los $55^{\circ} \mathrm{C}$ y en todos los casos el trabajar a esta temperatura aumenta su porcentaje de error. 
Los equipos disponibles en el mercado requieren afinar su sensibilidad para facilitar el monitoreo del cumplimiento de las normas y los límites máximos permisibles que estas imponen.

\section{A FUTURO}

La aplicación de SBC en calidad del aire se encuentra en innovación y desarrollo constante, los modelos comerciales disponibles se mejoran rápidamente. Latinoamérica se ha visto involucrada en este auge tanto en desarrollo como en opciones nuevas de implementación. En México, si bien se han utilizado los SBC para el monitoreo de calidad del aire con estaciones móviles que se encuentran disponibles de forma comercial, se encuentra en desarrollo un proyecto para el uso de estaciones compactas hibridas (meteorológicas-calidad del aire) utilizando esta tecnología en tiempo real y de forma ambulatoria (Figura 4).
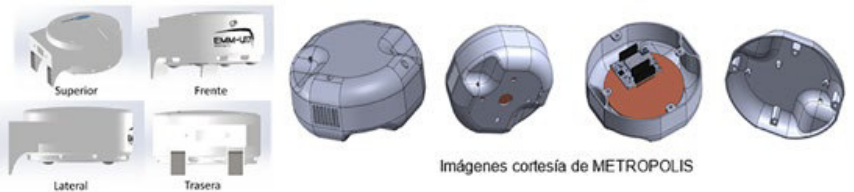

Figura 4. Prototipos de una estación compacta híbrida.

En el 2017 estas unidades ya fueron utilizadas en un monitoreo de prueba en la ciudad de Hermosillo, Sonora (Figura 5 y 6). El control de operación se realizó a tiempo real por medio de monitores, verificando las rutas de las unidades móviles.

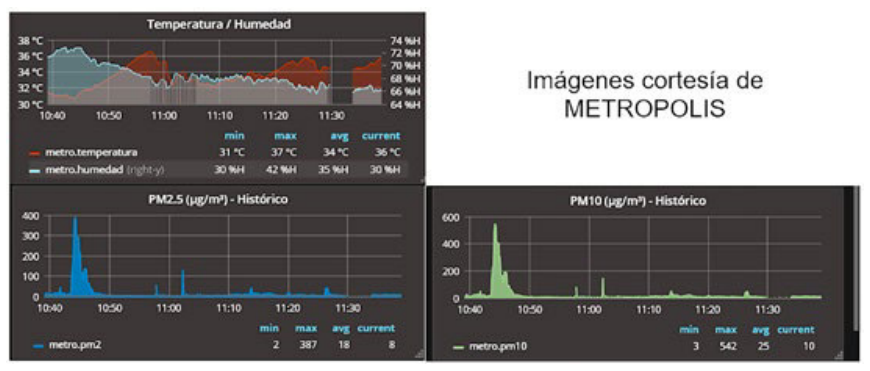

Figura 5. Graficas históricas de las variables de Temperatura, Humedad Relativa, $\mathrm{PM}_{10}$ y $\mathrm{PM}_{2.5}$.

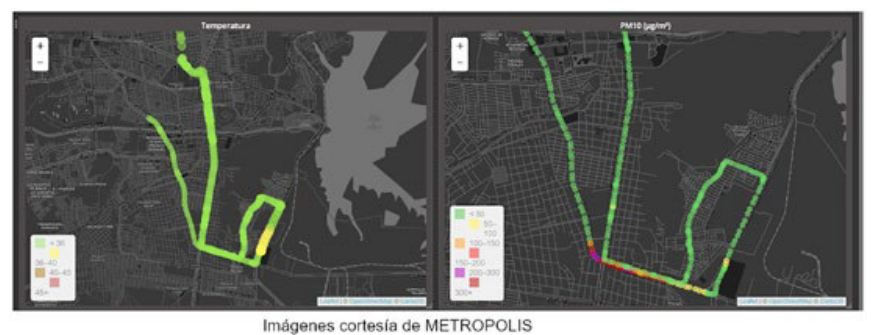

Figura 6. Mapeo de las variables de Temperatura y $\mathrm{PM}_{10}$.

\section{CONCLUSIONES}

El desarrollo de tecnología como la de los SBC para el monitoreo de la calidad del aire debe ser constante hasta lograr su optima aplicación.

Los modelos disponibles no cuentan con todas las características deseadas, pero son una base viable para comenzar la aplicación de estos dispositivos.

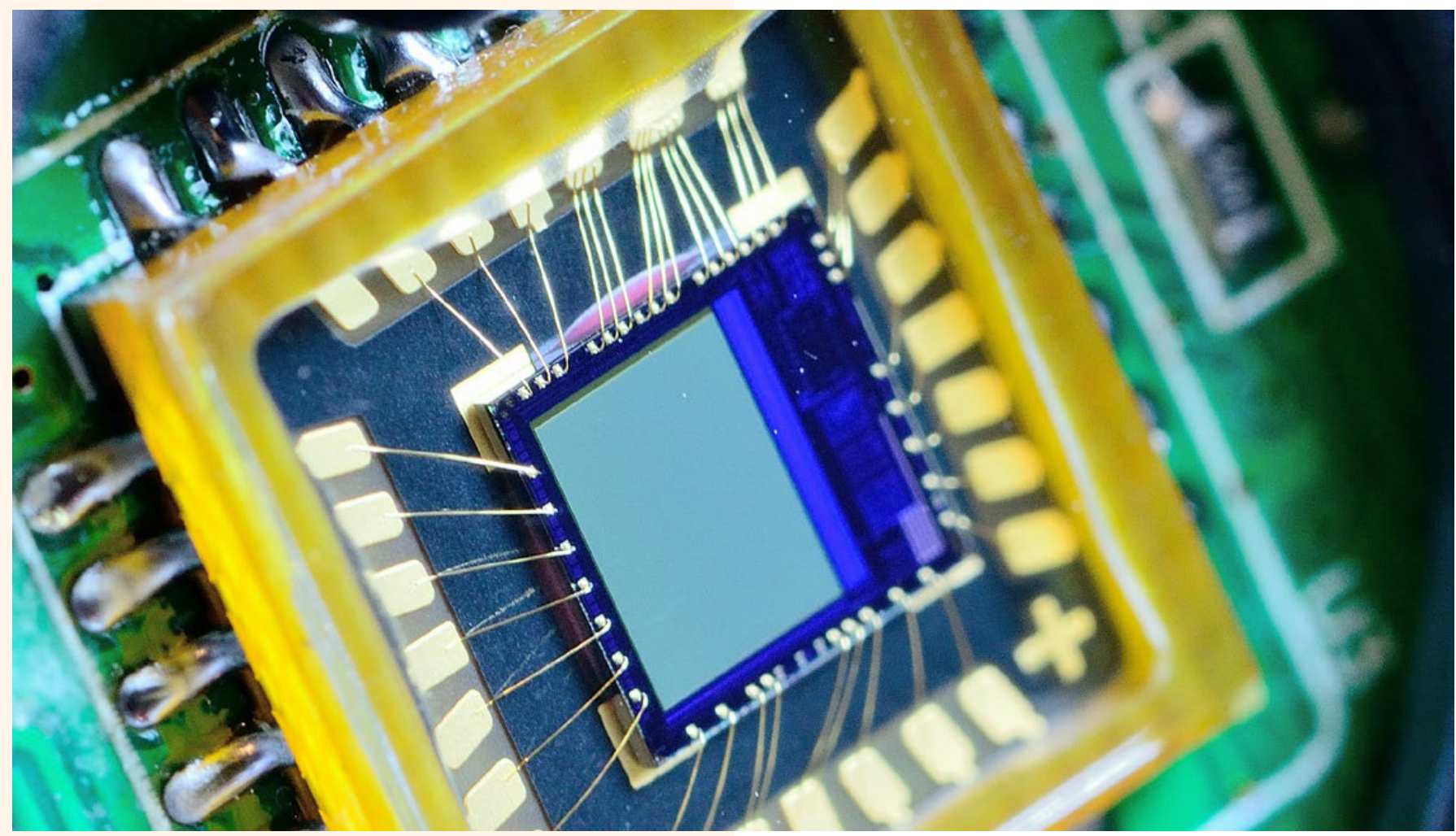


El interés de la comunidad científica en estos temas es evidente, y la necesidad de un mayor volumen de datos para la toma de decisiones permite abrir un horizonte a una mejora constante de estos dispositivos.

\section{BIBLIOGRAFÍA}

[1] R. Williams, T. Watkins y R. Long, "Findings from The 2013 EPA: Air Sensors Whorkshop", Air \& Waste Management Association, pp. 5-24, 2014.

[2] W. Jiao, G. Hagler y R. Williams, "Community Air Sensor Network (CARSENSE) Project: Lower cost, Continuous Ambient Monitoring Methods", EPA, Atlanta, Georgia USA, 2015.

[3] A. Luna, Á. Talavera y L. Cano, "Uso de sensores electroquímicos de bajo costo para el monitoreo de la calidad del aire en el distrito de San Isidro - Lima - Perú", Centro de Investigación Universidad del Pacífico (CIUP) , pp. 1-17, 2017.

[4] F. Pérez Maquieira, "Integración y Test de Módulos de Medición de Partículas PM2.5 y PM10 para la valoracion de la calidad del aire", Madrid : Universidad Politécnica de Madrid , 2018.

[5] SSA1-020, NOM-020-SSA1-2014, Diario Oficial de la Federación (DOF), Ciudad de México, 2014.

[6] SSA1-021, NOM-021-SSA1-1993, Diario Oficial de la Federación (DOF), Ciudad de México, 1994.

[7] SSA1-022, NOM-022-SSA1-2010, Diario OFicial de la Federación (DOF), Ciudad de México, 2010.

[8] SSA1-023, NOM-023-SSA1-1993, Diario Oficial de la Federación, Ciudad de México, 1994.

[9] COFEPRIS-025, NOM-025-SSA1-2014, DOF (Diario Oficial de la Federación), Ciudad de México, 2014.

[10]L. Escalona, L. Manganiello, M. López y C. Vega, "Los sensores químicos y su utilidad en el control de gases contaminantes", Revista Ingeniería UC, pp. 74-88, 2012.

[11]L. d. Valle, Programarfacil.com, Luis delValle, n/a n/a 2017. [En línea]. Available: https://programarfacil.com/podcast/82escoger-mejor-sensor-temperatura-arduino/.

[Último acceso: 29 Mayo 2018].

[12]MICROCHIP, Digi-Key.com, Digi-Key.com, [En línea]. Available: https://www.digikey.com.mx/es/product-highlight/m/ microchip-technology/mcp9902-remote-temperaturesensors. [Último acceso: 29 Mayo 2018].

[13]Artekit Labs, Artekit, Artekit, [En línea]. Available: https:// www.artekit.eu/products/breakout-boards/sensors/aksi7021/. [Último acceso: 11 Noviembre 2019].

[14]Hobby Electronics, Hobby Electronics, Hobby Electronics, [En línea]. Available: http://www.hobbytronics.co.uk/rht03humidity-temp-sensor. [Último acceso: 8 Octubre 2018].

[15]Electronilab, electronilab.co, Electronilab, [En línea]. Available: https://electronilab.co/tienda/sensor-de-presionbarometrica-bmp180/. [Último acceso: 6 Junio 2018].

[16]geekfactory, geekfactory.mx, geekfactory.mx, [En línea]. Available: https://www.geekfactory.mx/tienda/sensores/ sensor-de-presion-atmosferica-bosh-bmp085/. [Último acceso: 6 junio 2018].

[17]L. Ada, adafruit, adafruit, 31 Julio 2015. [En línea]. Available: https://learn.adafruit.com/adafruit-bmp280-barometricpressure-plus-temperature-sensor-breakout/pinouts. [Último acceso: 6 Junio 2018].

[18]Campbell Scientific, Inc., s.campbellsci.com, Abril 2017. [En línea]. Available: https://s.campbellsci.com/documents/us/ manuals/cs100.pdf. [último acceso: 6 Junio 2018].

[19]Campbell Scientific, Inc. , s.campbellsci.com, Julio 2019. [En línea]. Available: https://s.campbellsci.com/documents/us/ manuals/cs106.pdf. [Último acceso: 11 Noviembre 2019].

[20]e-radionica, softroboticstoolkit, [En línea]. Available: https://softroboticstoolkit.com/files/sorotoolkit/files/ mps20n0040d-s_datasheet.pdf. [Último acceso: 8 Octubre 2018].

[21]SHARP, Media.digikey.com, 11 noviembre 2018. [En línea]. Available: https://media.digikey.com/pdf/Data\%20Sheets/ Sharp\%20PDFs/GP2Y1010AU0F.pdf.

[22]TELAIRE,SmartDustSensorSW-PWM-01C,11 Noviembre2018. [En línea]. Available: file:///C:/Users/ke_cu/AppData/Local/ Packages/Microsoft.MicrosoftEdge_8wekyb3d8bbwe/ TempState/Downloads/AAS-930-185B-Telaire-SMART-DustSensor-090517-web\%20(1).pdf.

[23]Bjhike, Zeanoit, 11 Noviembre 2018. [En línea]. Available: https://www.zeanoit.jp/pdf/3f-SEN0177.pdf.

[24]SPEC, SPEC-SENSORS DGS-03, 11 noviembre 2018. [En línea]. Available: https://www.spec-sensors.com/wp-content/ uploads/2017/01/DGS-O3-968-042_9-6-17.pdf.

[25]SPEC, SPEC-SENSORS 3SP_O3_20, 11 Noviembre 2018. [En línea]. Available: https://www.spec-sensors.com/wpcontent/uploads/2016/02/3SP_O3_20-P-Package-110-406. pdf.

[26]SPEC, SPEC-SENSORS DGS-SO2 968-038, 11 Noviembre 2018. [En línea]. Available: https://www.spec-sensors.com/ wp-content/uploads/2017/01/DGS-SO2-968-038.pdf .

[27]ICON, gasdetectorshop.com, 11 Noviembre 2018. [En línea]. Available: https://www.gasdetectorshop.com/C03-0973100-RAE-Sulfur-dioxide-SO2-Sensor-p/c03-0973-100.htm.

[28]SPEC, SPEC-SENSORS 3SP_SO2_20 P Package110-601, 11 Noviembre 2018 . [En línea]. Available: https://www.specsensors.com/product/so2-sulfur-dioxide-sensor-20-ppmpinned-package/.

[29][29] SPEC, SPEC-SENSORS DGS-NO2, 11 Noviembre 2018. [En línea]. Available: https://www.spec-sensors.com/wpcontent/uploads/2017/01/DGS-NO2-968-043_9-6-17.pdf.

[30]Winsen, Alibaba, 11 Noviembre 2018. [En línea]. Available: https://spanish.alibaba.com/product-detail/gas-sensor-forarduino-gas-sensor-module-of-co-o2-nh3-h2s-no2-so2cl2-o3-60416554716.html.

[31]Alphasense, Directindustry, 11 Noviembre 2018. [En línea]. Available: http://www.directindustry.es/prod/alphasense/ product-16860-38596.html.

[32]SPEC, SPEC-SENSORS 3SP_CO_1000 Package 110-109, 11 Noviembre 2018. [En línea]. Available: http://www.specsensors.com/wp-content/uploads/2016/04/3SP_CO_1000C-Package-110-109.pdf.

[33]SPEC, SPEC-SENSORS 3SP_CO_1000 Package 110-102, 11 Noviembre 2018. [En línea]. Available: http://www.specsensors.com/wp-content/uploads/2016/04/3SP_CO_1000P-Package-110-102.pdf.

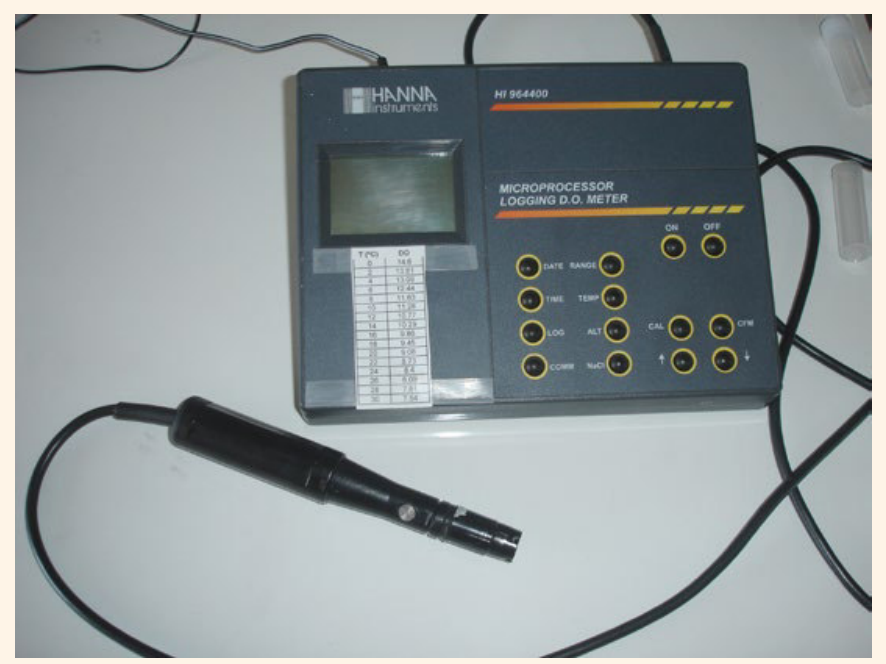

